\title{
Examining the Complex Relationship Between Greek Life and Alcohol: A Literature Review
}

\author{
Cherry Danielson \\ Simone Himbeault Taylor \\ Maureen Hartford
}

This literature review addresses the question of whether membership in a Greek organization contributes to the drinking behaviors of college students. Research findings are divided into these major categories: drinking frequency, motivation/reasons to drink, predicting Greek membership, culture of Greek life, perception bias, and consequences of drinking. Conclusions suggest that to be successful, intervention efforts must focus not only on individuals but also on organizational and institutional layers.

\section{Risky Drinking + Greeks - Responsibility = Tragic Event}

Not all alcohol-related tragedies on college campuses are connected to the Greek system. However, the perceptions of many within and outside academia place Greeks at the center of alcohol problems, especially binge drinking.

Cherry Danielson is a research associate at the University of Michigan, Simone Himbeault Taylor is the director of career planning and placement at the University of Michigan, and Maureen Hartford is president of Meredith College. 
At the 1998 NASPA Summit on Fraternity/Sorority Life in Dallas, higher education administrators and Greek life officials met to discuss issues currently facing the Greek system. Deaths of fraternity members from alcohol poisoning in Louisiana and Massachusetts were fresh in participants' minds. After intense discussion, this group concluded that the anecdotal information and personal experience driving most Greek life discussions needed to be replaced by fact or objective research. Summit participants posed the following research questions:

- Do Greeks drink more or more frequently than non-Greeks?

- Does the Greek system attract students who were heavy drinkers in high school?

- Does the Greek system change the drinking behavior of its members?

- Do Greek members on campuses accurately reflect their national organizations' stated values on alcohol?

A need emerged to examine critically these issues in light of available research. In response, the literature was reviewed.

\section{Literature Review}

The purpose of this review is to investigate the complex relationship between Greek life and alcohol. Although this is a circumscribed review, it is the authors' intention to approach these articles without a predetermined outcome. The following two questions guided this inquiry:

- Does membership in a Greek organization contribute to the drinking behaviors of college students?

- Is there a relationship between precollege drinking behaviors and Greek membership?

While many of the articles reviewed offer a broad range of information, we discovered the following major categories of research that provide a structure for this review: drinking frequency, motivation/ reasons to drink, predicting Greek membership, culture of Greek life, perception bias, and consequences of drinking. 
This review is not intended to offer solutions per se, but to close the gap between anecdotal information and fact by providing a systematic review of the research to date on this central issue. In turn, this review can provide practitioners with a tool to examine the situation on their own campus and possibly inform subsequent decisions regarding alcohol policies.

\section{Drinking Frequency}

Several studies document general drinking characteristics of college students. These studies indicate that $74 \%$ of students used alcohol prior to entering college (O'Connor, Cooper, \& Theil, 1996) and about 33.3\% engaged in binge drinking behaviors in high school (Wechsler, Downdall, Maenner, Gledhill-Hoyt, \& Lee, 1998). By the time students reach college, $84 \%$ report that they drank alcohol and the remaining $16 \%$ identify themselves nondrinkers. When asked about their frequency of drinking, approximately $70 \%$ of all students report drinking in a relatively infrequent and moderate manner primarily for social purposes and 14\% report heavy drinking patterns (Haworth-Hoeppner et al., 1989). Heavy drinking is defined by Haworth-Hoeppner et al. (1989), as those who drink once or twice a week with six or more drinks per occasion or five times a week with four to five drinks per occasion.

In the College Alcohol Study by Wechsler et al. (1998), findings indicate that the level of binge drinkers in the college student population between 1993 and 1997 remained at approximately 42.7\%, but the major change involved an increase in the proportion of abstainers from $15.6 \%$ to $19.0 \%$. Wechsler et al. (1998) define binge drinking as four or more drinks on an occasion for women and five or more for men. In the 1996 Core Alcohol and Drug Survey, 10\% of the students report they consumed 15 or more drinks on an average weekly basis, $38 \%$ of students identify themselves as abstainers, and another $13 \%$ report they consumed only one drink per week (Meilman, Presley \& Cashin, 1997). The noticeably dissimilar results in these two studies may be partially accounted for by operational definition differences.

While studies have investigated numerous facets of college drinking, one finding consistently emerges: fraternity members drink more frequently and more heavily than their nonfraternity peers (Alva, 1998; 
Cashin, Presley, \& Meilman, 1998; Faulkner, Alcorn, \& Garvin, 1989; Goodwin, 1992; Harrington, Brigham, \& Clayton, 1999; O'Connor et al., 1996). Research also shows that race and gender-specifically, white males-combine with fraternity membership as significant factors of heavy drinking (Faulkner et al., 1989; Goodwin, 1992; Haworth-Hoeppner et al., 1989; Wechsler et al., 1998; Werner \& Greene, 1992). Furthermore, these findings hold true even when body weight is taken into consideration (Goodwin, 1992).

Although there is a broad variance of drinking frequencies within both the Greek and non-Greek populations, it is well documented that there is a relationship between Greek membership and intensity of drinking (Engs \& Hanson; Engs, Kraft, \& Kaplan, cited by HaworthHoeppner et al., 1989). For example:

- $43.6 \%$ of non-Greek students, compared to $19.3 \%$ of fraternity and sorority members, reported no alcohol use in the past 30 days (Alva, 1998);

- $10 \%$ of the non-Greek students, compared to $20 \%$ of fraternity and sorority members, are heavy drinkers (Haworth-Hoeppner et al., 1989);

- as many as $47 \%$ of those residing in a Greek house reported themselves as heavy drinkers, compared to $14 \%$ among students at large (Haworth-Hoeppner et al., 1989).

According to Lo and Globetti (1995), compared to nonmembers, fraternity members not only drink more alcohol per occasion but also are three times more likely to change from a low to a high frequency of drinking as they move from high school to college. Sorority members are five times more likely than are nonmembers to exhibit this change.

\section{Motivation/Reasons to Drink}

Knowing the frequency of drinking does not provide sufficient information, asserts Klein (1992). He states it is important to understand the subjective aspect of why students drink because this affects their alcohol patterns. Spindler's (1964) research describes one group of reasons as socially acceptable, positive in nature, and associated with moderate, nonproblematic alcohol use (cited in Klein, 1992). In Klein's empirical study, these reasons are cited by the majority of 
respondents (Klein, 1992), and might also reflect the motivations of $70 \%$ of college students who call themselves infrequent and moderate drinkers in Haworth-Hoeppner's (1989) study.

In the student population at large, common reasons cited to drink were peer pressure and what we derive to be a sort of "social courage," whereby alcohol is used to ease dating tension and social shyness. Students who drink moderately seem to "drink when the social setting warrants it, but typically not with a goal in mind" (Klein, 1992, p. 26). In contrast, "problem drinkers . . . are less likely to drink for social reasons and more likely to drink to escape" (Canterbury, 1990, p. 8). Additionally, students who drink most heavily tend to have rather well defined rationales that indicate a reliance on alcohol (Klein, 1992).

Klein's (1992) study includes comparison data indicating that fraternity house residents are significantly more likely to cite reasons that correlate with heavy drinking and indicate a reliance on drinking. Further building on this theme, male Greek members who are residents of fraternity houses cited the most reasons to drink and are the only group who cite negative or disintegrative reasons for drinking. These results corroborate other studies that demonstrate greater consumption of alcohol and higher levels of alcohol abuse among members of Greek organizations than their non-Greek peers (Cashin et al., 1998; Klein, 1992). The question of the socializing effects of the fraternity environment is addressed later in this review.

\section{Predicting Greek Membership}

Although the literature in this review indicates that both frequency of and motivations for drinking are differentiated by Greek membership, establishing the relationship does not speak to the issue of causality. Specifically, the following studies respond to the question, "Is a more excessive drinking pattern an antecedent or a consequence of Greek membership?" (Lo \& Globetti, 1995, p. 1315).

Precollege alcohol use has been the focus of a limited number of studies over the past decade. Nevertheless, the sparse but significant results demonstrate that the extent of high school drinking is a major predictor of college drinking, which suggests that previous experience continues to shape future practice (Goodwin, 1992). Moreover, stu- 
dents who drink relatively frequently, in higher quantity, and who experience alcohol-related problems while in high school are more likely than others to join a fraternity or sorority in college (Lo \& Globetti, 1995; O'Connor et al., 1996; Werner \& Greene, 1992). Werner and Greene's (1992) study shows that 93\% of those students reporting frequent drinking and $89 \%$ of those reporting frequent bingeing expressed an intent to join a fraternity or sorority. Thus, this literature suggests that, at a minimum, a more excessive high school drinking pattern is associated with and is a predictive element of Greek affiliation.

\section{Culture of Greek Life}

Literature suggests a complex model to understand the contribution of the Greek life culture to drinking behaviors of its members. To address the question of influence, studies have investigated the power of norms, attitudes, and behaviors of the Greek life environment. Fraternities and sororities have a reputation with regard to drinking. "Students appear to arrive at college with the expectation that the Greek system will support a heavy drinking pattern" (Werner \& Greene, 1992, p. 491). While most studies define problem drinking using direct information about drinking behaviors, in their empirical study, Werner and Greene include a definition of problem drinking based on beliefs and attitudes. Although this model only indirectly measures drinking patterns, "beliefs about the effects that drinking will produce are known to influence both consumption and the subjective experience of intoxication" (Marlatt $\&$ Rohsenow; Sher, cited by Werner \& Greene, 1992, p. 490). According to Werner and Greene's (1992) findings, when the socialization of Greek affiliation reinforces those beliefs and attitudes, this translates into high-risk drinking behaviors.

In the fraternity environment where higher levels of drinking behaviors exist, motivations for drinking are strategically cultivated through a complex socialization process that will be discussed later in this review (Faulkner, 1989; Kuh, 1993, Lo \& Globetti, 1995). Based on empirical research, Werner and Greene (1992) argue that "heavy alcohol consumption among fraternity pledges is strongly correlated with a positive view of the socialization value of alcohol" (p. 487). For these 
students, the expectation of the benefits of drinking provides motivation to engage in drinking behaviors.

Many of the studies reflect the "increasingly important role that alcohol consumption is perceived to have in the social dynamics of fraternity life" (Faulkner et al., 1989; Lo \& Globetti, 1995). Studies demonstrate that the Greek culture not only intricately incorporates a value of heavy drinking (Kuh, 1993; O'Connor et al., 1996) but also uses alcohol to entice, reward, reinforce, and maintain behaviors among Greek membership. In fact, Leavy (1979) describes this socialization process within a fraternity as a "form of education in which the subject is drinking and the classroom is the fraternity house" (cited in O'Connor et al., 1996, p. 669). Larimer, Irvine, Kilmer, and Marlatt (1997) use the social learning theory to explain the "role of the specific organizational norms in creating a high-risk environment for alcohol use by students residing in some Greek organizations" (p. 595). They point to evidence of social learning in the new members' strong modeling behavior of the house drinking norms.

Kuh and Arnold (1993) utilized the ethnological tool of a cultural audit to probe the culture within a Greek fraternity. Their findings offer qualitative insight into the socialization of pledges and the replication of beliefs, values, and attitudes among members. Results indicate that "alcohol is a key element in a complicated system of rewards and sanctions used by Greek organizations to socialize newcomers" (Kuh \& Arnold, 1993, p. 34).

Kuh and Arnold (1993) explain their findings using three interrelated cultural layers: artifacts, strategic perspectives and values, and assumptions and beliefs. They discovered evidence in all three layers that alcohol plays a prominent role in Greek life. Cultural artifacts include advertisements for alcohol, posters, and alcohol itself that is used to decorate personal and shared space. These researcher-observers noted that social functions are visibly about alcohol and conversations among members are "peppered" with language and experiences relating to alcohol (Kuh \& Arnold, 1993).

Kuh and Arnold (1993) point out, in terms of strategic perspectives and values, that norms and fundamental "oughts" are determined by influential members of the fraternity. Additionally, in contrast to espoused 
intellectual and humanitarian values of Greek organizations, these researchers observed that students' behaviors overwhelmingly reflect hedonistic, anti-intellectual behaviors and attitudes, and the value of alcohol in the member's life.

Examining assumptions and beliefs reveals that fraternity members share an organizationally constructed reality. Kuh and Arnold (1993) find that members of fraternities share the self-perception that they are "special" and can "do whatever [they] want as long as no one beyond the group knows or is directly affected" (p. 331). Fraternity members believe that their behavior is not governed by the normal institutional expectations and policies or even civil law, but "the ultimate authority in determining whether behavior is acceptable or valued is the group itself" (1993, p. 332).

Developing these norms, beliefs, and behaviors in new pledges is the purpose of the socialization process. Once in full membership, individuals do not question the practices and values of the organization. The fraternities strongly defend themselves from both internal and external threats through a fierce sense of loyalty and strong, influential alumni (Kuh \& Arnold, 1993).

\section{Perception Bias}

Studies show that college students in general have a biased perception of the frequency and quantity of alcohol consumption by their peers (Baer \& Carney, 1993). Additionally, Baer \& Carney argue that "beliefs about what is 'normal' or 'typical' for alcohol consumption may influence both behavior and attitudes about drinking within social groups"(1993, p. 54).

When assessing the drinking patterns of individual college students and their perception of the drinking patterns of close friends and reference groups, virtually all students report that their friends drink more than they do (Baer, Stacy, \& Larimer, 1991; Baer \& Carney, 1993; Larimer et al., 1997). These biases are particularly evident within fraternities and sororities (Baer et al., 1991).

Some researchers explain this perception bias using the model of the Fundamental Attribution (Baer \& Carney, 1993). This theory states 
that people are more likely to attribute the behavior of others to dispositional characteristics and yet see their own behavior as situationally dependent. In their study, Baer \& Carney (1993) find that subjects define the same behavior of someone else as more indicative of problem drinking than of themselves.

Baer \& Carney (1993) suggest that these exaggerations represent flawed information processing. They caution that such beliefs may serve to make problem drinking appear less risky and pose an order of resistance to prevention efforts. The implication is that because of these false perceptions, college students who see themselves as behaving normatively within their fraternity or sorority may lack the cues to alert them to signs of risk and potential problems.

\section{Consequences of Drinking}

Research regarding drinking patterns might not attract much interest if there were no risks or consequences associated with these behaviors. Unfortunately, there are negative outcomes associated with alcohol consumption. Gliksman, (1988) grouped such problems into five categories: personal effects, sociallegal effects, drinking and driving, major school problems, and minor school problems. Corroborating other research (Klein, 1989), Gliksman's findings also indicated overall significant differences between males and females in two ways. First, "males experienced more of the consequences of alcohol use than females"(1988, p. 1287); and second, "female students reported an increase in problems during their first year of college only in the areas of personal effects and major school problems, while male students reported increases in problems in all categories except Drinking and Driving" (1988, p. 1293). Because research tends to focus on socially disruptive, negative consequences that are more likely to be experienced by men, there is insufficient understanding of the "less visible, drinking-related negative consequences more frequently experienced by women; e.g., depression, damaged interpersonal relationship, unwanted sexual experiences or pregnancy" (Berkowitz \& Perkins, 1987 , p. 126) and bulimia (Meilman, von Hippel, \& Gaylor, 1991).

Results from both the 1993 and 1997 College Alcohol Studies show that the consequences related to alcohol occur at much higher rates among occasional or frequent binge drinkers than nonbinge drinkers 
(Wechsler et al., 1998). These researchers find that occasional binge drinkers are 5 times more likely to experience 5 or more alcohol-related problems and that frequent binge drinkers are 22 times as likely to do so (Wechsler et al., 1998).

In a sample of only Greek members, Goodwin (1992) connects behaviors of drinking and drug use with physical tolerance. Findings demonstrate that students use their own physical experiences to help them judge how concerned they should be about their drinking behaviors. Goodwin (1992) suggests that this type of self-assessment is an unreliable mechanism.

With ninety-eight percent of fraternity/sorority members drinking some quantity of alcohol every week, Goodwin (1992) declares that alcohol is clearly their drug of choice. Furthermore, these students show a higher incidence of alcohol-related problem behaviors than do their non-Greek peers (Cashin et al., 1998; Klein, 1992; Lichtenfeld, 1994). Thus, a profile of a group at risk emerges from the literature. "Men who are Greek organization members and fraternity house residents are at the greatest risk for suffering from the adverse consequences of their drinking behavior" (Klein, 1989, p. 326). Ironically, the same students who experience more problems are also less likely to take advantage of resources available for substance abuse issues (Klein, 1989).

Although studies demonstrate a relationship between drinking and grades (Goodwin, 1992; Klein, 1989), it is not clear if causal implications can be made. Students with lower grades may drink more than those with higher grades and it is also true that lower grades might result from drinking more (Goodwin, 1989). Clearly, we must be cautious when interpreting causality for problems associated with drinking behaviors.

Canterbury et al. (1990) studied psychosocial features of problem drinkers. Their findings indicated that, compared to nonproblem drinkers, problem drinkers "have poor impulse control, manifest deviant behavior, value academic success less and strive for independence" (Canterbury, 1990, p. 8). These researchers also found that "frequent heavy drinkers and students with psychosocial problems 
appeared disproportionately among students planning to join fraternities and sororities" (Canterbury, 1990, p. 2).

\section{Discussion}

The results of the reviewed studies present a complex picture of the relationship between alcohol and Greek membership. Together, the findings alert us to a campus subculture that is significantly different from the general college student population in which drinking attitudes and behaviors are embedded in the physical, cognitive, emotional, and cultural aspects of students' lives.

This relationship involves, on one hand, the predictability of Greek membership based on high school drinking and partying frequency (Lo \& Globetti, 1995; O'Connor et al., 1996; Werner \& Greene, 1992) and, on the other hand, the strategic socialization of Greek pledges to value drinking and perpetuate its significance within the Greek culture (Kuh, 1993). Additionally, factors contributing to increased risk involve faulty perceptions of normative behavior (Baer et al., 1991; Baer \& Carney, 1993) and defective self-assessment mechanisms (Goodwin, 1992). Subsequently, this melange does not demonstrate a simple cause and effect. While the Greek culture provides opportunity and a permissive environment, there is a subset of high school students who come to college with a receptivity, if not a desire, for this culture.

At this juncture the practitioner may ask "So, what should we be doing about this situation?" While a separate body of literature exists around interventions, the results are difficult to generalize broadly to other institutions because of the campus-specific nature of interventions. In practice, designing appropriate interventions for an institution is not a simple issue and solutions are likely to be complex and multilayered. In addition, much of this literature tends to be best-practice writings that are not necessarily research-based. The consensus of the literature is that the process of developing interventions is an institutionally focused task that by virtue of the issue itself must be embedded in each unique campus culture and situation. While specific interventions are not discussed here, many researchers have made recommendations about important factors to consider in the development of 
programming (Alva, 1998; Baer et al., 1991; Berkowitz \& Perkins, 1987; Canterbury et al., 1990; Cashin et al., 1998; Faulkner et al., 1989; Goodwin, 1989, 1992; Harrington et al., 1999; Klein, 1989; Kuh, 1993; Larimer et al., 1997; Lo \& Globetti, 1995; Werner \& Greene, 1992). The considerations can be grouped into three categories: the individual, organizational, and institutional layers.

At the individual level, research suggests that interventions should target first-year students and heavy drinkers in particular because they are in the greatest need (Alva, 1998; Goodwin, 1989). Additionally, efforts to change behavior should address inaccurate beliefs of individuals about normative drinking and drug use (Baer et al., 1991) and attend to psychosocial features that predispose to alcohol abuse and addiction (Canterbury et al., 1990).

As evidenced in the description of the Greek life culture, there are organizational-level considerations that are key to changing the pattern of drinking behaviors among members. For example, Goodwin (1989) recommends working with a nucleus of those Greek members who display moderate consumption in order to institutionalize an effort to decrease alcohol consumption and the acceptability of inebriation in social situations (Goodwin, 1989). Researchers also recommend the creation of programs designed to modify peer norms that include individual and group attitudes, beliefs, and expectations surrounding the social advantages of drinking (Alva, 1998; Cashin et al., 1998; Faulkner et al., 1989; Goodwin, 1989; Harrington et al., 1999; Larimer et al., 1997). Such modifications of Greek systems require training of Greek life staff and membership to become "sensitive to the drinking habits of their pledges and subsequently examine their role as an 'enabler' of already-established drinking habits" (Lo \& Globetti, 1995, p. 1320). The strength of organizationally focused interventions lies in the ability to hold local chapters responsible for bringing about cultural change (Kuh, 1993).

The individual and organizational levels of programming require institutional commitment to bring about complex change. Researchers suggest that campuses should use student health services to routinely screen for problem drinking (Werner \& Greene, 1992, p.491) and implement programs that combine educational and regulatory approaches in the campus community (Alva, 1998). Regular profes- 
sional development should assist student affairs professionals to become cultural practitioners who understand cultural perspectives and culture-change strategies (Kuh, 1993, p.333). Staff members also need to gain a greater awareness of gender differences among students, including differential consequences of drinking and transition-related needs (Berkowitz \& Perkins, 1987). Finally, the institution might opt to defer "rush" until the final month of the first year of college or the second year, or ultimately eliminate/sanction groups that are unwilling to modify their behaviors and culture (Kuh, 1993).

To incorporate this range of considerations into the fabric of interventions and programming suggests a holistic perspective. As such, each campus must evaluate its own situation to customize programs in light of sound research results. Institutions actively engaged in drinkingmodification programs might also benefit from ongoing evaluation of the outcomes of such programs; we can easily err by making an assumption of "rationality." As an example: for years, educators believed that if we provided well-reasoned information about alcohol usage to students, appropriate behavior would follow.

In conclusion, the literature clearly argues for research exploring foundational theories that offer perspective on Greek life and alcohol, and encourages practitioners to avail themselves of research findings in an effort to link interventions to those results. In turn, given the complex nature of this topic, practitioners and policy makers should be cautious of studies that are too narrowly focused or simplistically interpreted. As reviewers of the literature, we challenge those concerned about Greek life to conduct meta-trend analyses of Greek/alcohol studies to understand the complex relationships among factors leading to problem drinking among Greek membership. Because of the invested interest of both the national Greek organizations and individual institutions, collaboration may be necessary to produce thorough studies; combined efforts, from design to interpretation, may provide a greater perception of neutral and credible research. Lastly, research on Greek life and alcohol should play a larger role in informing practice. 


\section{References}

Alva, S. A. (1998). Self-reported alcohol use of college fraternity and sorority members. Journal of College Student Development, 39 (1), 3-10.

Baer, J. S., Stacy, A., \& Larimer, M. (1991). Biases in the perception of drinking norms among college students. Journal of Studies on Alcohol, 52 (6), 580-586.

Baer, J. S., \& Carney, M. M. (1993, January). Biases in the perceptions of the consequences of alcohol use among college students. Journal of Studies on Alcohol, 54, 54-60.

Berkowitz, A. D., \& Perkins, H. W. (1987, September). Recent research on gender differences in collegiate alcohol use. Journal of American College Health, 36, 123-129.

Canterbury, R. J., Gressard, C. F, Vieweg, W. V. R., Grossman, S. J., Westerman, P. S., \& McKelway, R. B. (1990). Psychosocial inventory among first-year college students by patterns of alcohol use. Advances in Alcohol \& Substance Abuse, 9 (3/4), 1-11.

Cashin, J. R., Presley, C. A., \& Meilman, P. W. (1998, January). Alcohol use in the Greek system: Follow the leader? Journal of Studies on Alcohol, 59 (1) 63-70.

Faulkner, K. K., Alcorn, J. D., \& Garvin, R. B. (1989). Prediction of alcohol consumption among fraternity pledges. Journal of Alcohol $\&$ Drug Education, 34, 12-20.

Gliksman, L. (1988). Consequences of alcohol use: Behavior changes and problems during first year of university. The International Journal of the Addictions, 23 (12), 1281-1295.

Goodwin, L. (1989, September). Explaining alcohol consumption and related experiences among fraternity and sorority members. Journal of College Student Development, 30, 448-458.

Goodwin, L. (1992, Winter). Alcohol and drug use in fraternities and sororities. Journal of Alcohol \& Drug Education, 37 (2), 52-63.

Harrington, N. G., Brigham, N. L. \& Clayton, R. R. (1999, July). Alcohol risk reduction for fraternity and sorority members. Journal of Studies on Alcohol, 60 (4), 521-527.

Haworth-Hoeppner, S., Globetti, G., Stern, J., \& Morasco, F. (1989). The quantity and frequency of drinking among undergraduates at a southern university. The International Journal of the Addictions, 24 (9), 829-857. 
Klein, H. (1992, Winter). Self-reported reasons for why college students drink. Journal of Alcohol and Drug Education, 37 (2), 14-28.

Klein, H. (1989, July). Helping the college student problem drinker. Journal of College Student Development, 30, 323-331.

Kuh, G. D. A., J.C. (1993, September). Liquid bonding: A cultural analysis of the role of alcohol in fraternity pledgeship. Journal of College Student Development, 34, 327-334.

Larimer, M. E., Irvine, D. L., Kilmer, J. R., \& Marlatt, G. A. (1997, November). College drinking and the Greek system: Examining the role of perceived norms for high-risk behavior. Journal of College Student Development, 38 (6), 587-598.

Lichtenfeld, M. K., W. A. (1994). Factors in college students' drinking. Psychological Reports, 74, 927-930.

Lo, C. C., \& Globetti, G. (1995). The facilitating and enhancing roles Greek associations play in college drinking. The International Journal of the Addictions, 30 (10), 1311-1322.

Meilman, P. W., von Hippel, F. A., \& Gaylor, M. S. (1991, July). Self-induced vomiting in college women: Its relation to eating, alcohol use, and Greek life. The Journal of American College Health, 40, 39-41.

Meilman, P. W., Presley, C. A., \& Cashin, J. R. (1997, March). Average weekly alcohol consumption: Drinking percentiles for American college students. The Journal of American College Health, 45, 201-204.

O'Connor, R.M., Jr., Cooper, S.E., \& Thiel, W.S. (1996). Alcohol use as a predictor of potential fraternity membership. Journal of College Student Development, 37 (6), 669-675.

Smith, D. S., Collins, M., Kriesberg, J. P., Volpicelli, J. R., \& Afterman, A. I. (1987, September). Screening for problem drinking in college freshmen. The Journal of American College Health, 36, 89-94.

Wechsler, H., Downdall, G. W., Maenner, G., Gledhill-Hoyt, J., \& Lee, H. (1998, September). Changes in binge drinking and related problems among American college students between 1993 and 1997. Journal of American College Health, 47, 57 - 68.

Werner, M.J., \& Greene, J.W. (1992). Problem drinking among college freshmen. Journal of Adolescent Health, 13 (6), 487-492. 